\title{
DEFORMATION RESPONSE OF POLYDIMETHYLSILOXANE SUBSTRATES SUBJECTED TO UNIAXIAL QUASI-STATIC LOADING
}

\author{
Vladimír VinARSkÝ $^{a, *}$, FABiAna MARTino ${ }^{a}$, GiAnCARlo Forte $^{a}$, \\ JAN ŠLEICHRT ${ }^{b}$, VÁClAV RADA ${ }^{b}$, DANIEL KYTÝ $\check{R}^{b}$ \\ ${ }^{a}$ St. Anne's University Hospital Brno, International Clinical Research Center, Pekařká 53, 65691 Brno, Czech \\ Republic \\ ${ }^{b}$ Czech Academy of Sciences, Institute of Theoretical and Applied Mechanics, Prosecká 809/76, 19000 Prague 9, \\ Czech Republic \\ * corresponding author: vladimir.vinarsky@fnusa.cz
}

\begin{abstract}
To investigate cellular response of cardiomyocytes to substrate mechanics, biocompatible material with stiffness in physiological range is needed. PDMS based material is used for construction of microfluidic organ on chip devices for cell culture due to ease of device preparation, bonding, and possibility of surface functionalization. However it has stiffness orders of magnitude out of physiological range. Therefore, we adapted recently available protocol aiming to prepare substrates which offer stiffness in physiological range $5-100 \mathrm{kPa}$ using various mixtures of Sylgard. An in-house developed loading device with single micron position tracking accuracy and sub-micron position sensitivity was adapted for this experimental campaign. All batches of the samples were subjected to uniaxial loading. During quasi-static experiment the samples were compressed to minimally $40 \%$ deformation. The results are represented in the form of stress-strain curves calculated from the acquired force and displacement data and elastic moduli are estimated.
\end{abstract}

KEYworDs: Quasi-static loading, hyperelasticity, polydimethylsiloxane substrates, in-situ loading device.

\section{INTRODUCTION}

Mechanical stimulus emerged as important factor for cell and organ development, homeostasis, and disease in vivo. To investigate effects of static and dynamic mechanical stimulation of in vitro grown cells two different approaches are being currently used. To determine the effect of material stiffness in static conditions polyacrylamide (PA) gels which can be prepared in the range of stiffness reflecting in vivo situation are used. On the other hand, effects of dynamic mechanical stimulation (stretching) are investigated using polydimethylsiloxane (PDMS) based microfluidic devices, where cultivation membrane is orders of magnitude stiffer than what can be found in vivo. So far it has been shown that both static and dynamic mechanical stimuli influence cardiomyocyte specification and maturation in vitro.

In order to integrate physiological stiffness and possibility to actuate (stretch) the cultivation surface in one device we aimed to prepare PDMS based material with stiffness in physiological range, which can be used in pneumatically actuated microfluidic device and test its biocompatibility. As the actuation of the cultivation membrane in general changes material properties we decided to measure the stiffness with tensile strain up to $20 \%$ of original length to mimic its application in microfluidic device.

Biomechanical analysis of PDMS is challenging ex- perimental procedure because of very low material stiffness. There are two basic approaches for this purpose. For the micromechanical tested an atomic force microscopy or dynamic nanoindentation [1] could be used. This experimental procedure is very precise but for bulk material testing macromechanical approach is more appropriate to obtain overall material properties [2]. Moreover customised precise macroscopic loading experiments coupled with radiography imaging techniques could provide the information about internal microstructure changes during the loading [3]. For this purpose the material properties of PDMS mixtures was obtained employing fully customised experimental system.

\section{MATERIALS AND METHODS}

\subsection{SAMPLE PREPARATION}

Based on previously published data (see [4]) different ratios of Sylgard 184 and Sylgard 527 were mixed to obtain stiffness ranging from $5 \mathrm{kPa}$ up to $170 \mathrm{kPa}$. In order to further decrease stiffness we manipulated the ratio of components A and B of Sylgard 527 from 1.2 to 1 . The resulting mixtures were degassed, poured in desired vessels and polymerized at $65^{\circ} \mathrm{C}$ overnight.

The bulk material was polymerized in plastic test tubes with nominal inner diameter $12.00 \mathrm{~mm}$. Properly polymerized cylinders with diameter $12.00 \pm$ $0.05 \mathrm{~mm}$ were carefully removed and carve up to the 


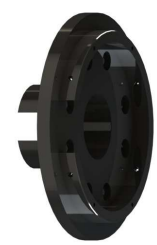

1

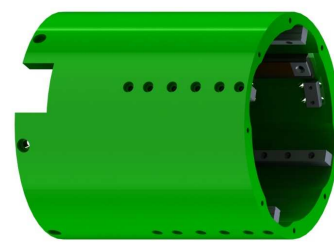

2

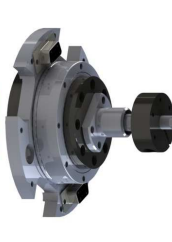

3

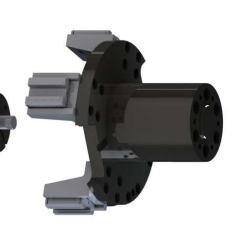

4

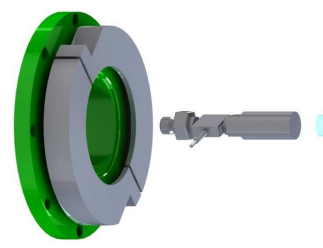

5
6

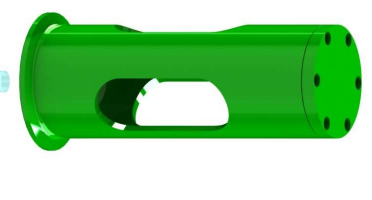

7

FiguRE 1. Visualization of the disassembled loading device with the main components: 1 - base plate (for rotary table), 2 - loading device frame, 3 - motor assembly with harmony gearbox, 4 - linear guide assembly, 5 - main body cover with clamps, 6 - loadcell and loading plate, 7 - loading chamber with loading plate

samples with height $14-16 \mathrm{~mm}$ using the microtome blade. Plan-parallel faces of the samples are required for relevant testing, therefore only the sampled with height precision $\pm 0.10 \mathrm{~mm}$ were selected for the testing. Precise height of the samples was derived from loading plates positions at the contact force $0.005 \mathrm{~N}$ and $0.010 \mathrm{~N}$ for load cells with nominal capacity $1 \mathrm{~N}$ and $10 \mathrm{~N}$ respectively. For the strain calculation the height was rounded to $50 \mu \mathrm{m}$.

\subsection{INSTRUMENTATION}

Estimated high compliance of the material require to use highly precise measuring procedure. Therefore, an in-house developed loading device originally intended for 4D microCT in detail description in [5] was employed. The device allows to perform analysis of biological samples and artificial product of tissue engineering in controlled ambient conditions. The design of compact table loading device allow to perform the mechanical testing up to $3 \mathrm{kN}$ with single micron position tracking. Using the magnetic linear encoder with nominal resolution $250 \mathrm{~nm}$ the device operates with sub-micron position sensitivity. Using the load cells with appropriate capacity, in this case miniature S beam load cells (LSB200 series, FUTEK Inc., USA) with nominal capacity $10 \mathrm{~N}$ and $1 \mathrm{~N}$ respectively, allows to provide reliable deformation analysis of the materials with elastic modulus in range of single $\mathrm{kPa}$. Main components of the loading device are depicted in Fig. 1.

For this purpose the loading frame was made of a high strength aluminium alloy (EN-AW-6082-T6) tube with $60 \mathrm{~mm}$ diameter with holes for sample manipulation and optional optical imaging. The loading plates made from the same material with diameter $16 \mathrm{~mm}$ ensure the proper support of the faces of the sample under high $(\approx 50 \%)$ deformation.

The motion of the system and all other peripherals are controlled using a custom developed control software based on the open-source project LinuxCNC running on the real-time kernel [6]. The control hardware consists of high-performance motion control com- ponents (MESA Electronics, U.S.A.), sensor readout electronics (LabJack, U.S.A.) and custom electronics.

\subsection{Experimental Procedure}

To obtain general information about the deformation behaviour of the mixtures and confrontation with findings presented in 4] set of pilot test was performed using 1 to 2 and 1 to 9 (Sylgard 184 to Sylgard 527) mixtures. It was found that i) the behaviour of the material is fully hyperelastic to minimally $60 \%$ strain ii) the sample is collapsing by sudden outsplashing at level of $70-80 \%$ compressive strain iii) the stress-strain behaviour during both loading and unloading phase was strain rate independent on level $0.005-0.05 \mathrm{~s}^{-1}$. Moreover, the samples due its very low stiffness exhibits high adhesion on the surfaces. It allows to measure tensile behaviour beyond the zero point at unloading of the compressed samples nearly to $10 \%$ tensile strain without any special sample fixation. Finally one sample of each type was stored in the fridge with constant temperature of $5^{\circ} \mathrm{C}$ the other were stored in laboratory temperature of $20-25^{\circ} \mathrm{C}$. Mechanical tests were performed on all samples at as delivered fresh state, after one week and after six weeks. No significant/measurable changes caused aging and/or storage condition were observed.

Based on the results of pilot test following experimental procedure was applied on all types of the samples. From each type of material three samples were measured. Each sample was put to loading chamber and its height was measured by the indicated contact. From this initial point the sample was compressed to the level of $40 \%$ strain on loading rate $20 \mu \mathrm{m} \cdot \mathrm{s}^{-1}$ (strain rate $\approx 0.015 \mathrm{~s}^{-1}$ ) and hold on for $5 \mathrm{~s}$ at this position. Then with the same loading rate the sample was continually unloaded and stretched to $10 \%$. From this point an other two loading cycled was performed. For the stress-strain analysis and elastic modulus estimation the average unloading curve was calculated.

\subsection{Data Evaluation}

Fully automatic evaluation procedure was developed using MATLAB (MathWorks Inc., USA). Primary 


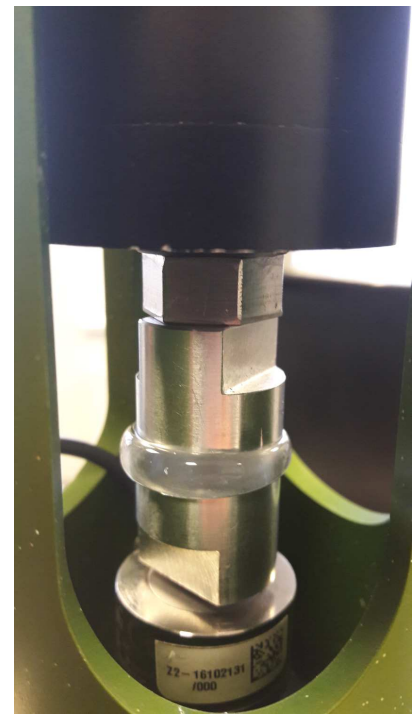

Figure 2. Detail of the compressed pure Sylgard 184 sample under $60 \%$ deformation in loading chamber

data acquired by control software with sampling frequency $200 \mathrm{~Hz}$ was imported and force log from load cell and position from encoder was post processed. From the record the unloading phases were extracted and averaged to one unloading force-displacement behaviour. For deformation behavior the stress $\sigma$ in all experimental analysis was considered as engineering stress obtained using

$$
\sigma_{\mathrm{eng}}=\frac{F}{A_{\mathrm{c}}}
$$

where Ac is cross-sectional area of the specimen calculated from minimal sample diameter measured before deformation. For the purpose of stress calculations, samples were considered ideally cylindrical, neglecting all geometrical irregularities. Engineering strain $\epsilon_{\text {eng }}$ was calculated by formula

$$
\epsilon_{\mathrm{eng}}=\frac{u}{h_{0}}
$$

where $u$ represents displacement measured by encoder and $h_{0}$ is height of the sample before the mechanical loading.

Magnitude of the Young's modulus at level of $0 \%$, $10 \%$, and $30 \%$ strain labeled $E_{0.0}, E_{0.1}$ and $E_{0.3}$ in stress-strain diagrams (see Figs. 3 (8) was estimated linear regression from 600 stress-strain values. The linear fit provide less than $0.5 \%$ error. Finally the GNUplot (command-line open source program for twoand three-dimensional data and function graphical visualization) code was generated and executed.

\section{Results}

Material properties and deformation behaviour of twelve different PDMS mixtures were derived from set of uniaxial loading tests. Each type of material was tested with the batch of the three samples. Stressstrain diagram of selected six clinically relevant mixtures together with Young's modulus are depicted and listed in Figs. 3,8 Light red stripe represents an envelope of measured data from whole batch. Dark red curve represents mean values.

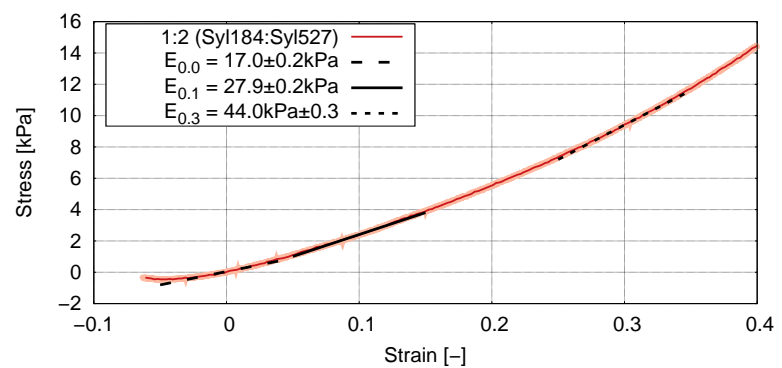

Figure 3. Deformation behaviour of 1 to 2 (Sylgard 184 to Sylgard 527) mixture

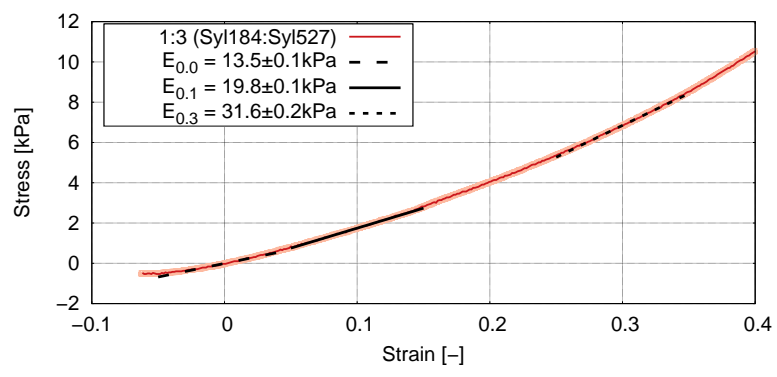

FiguRE 4. Deformation behaviour of 1 to 3 (Sylgard 184 to Sylgard 527) mixture

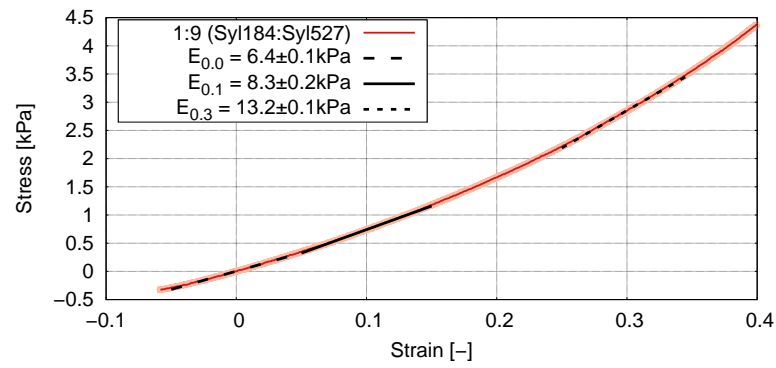

Figure 5. Deformation behaviour of 1 to 9 (Sylgard 184 to Sylgard 527) mixture

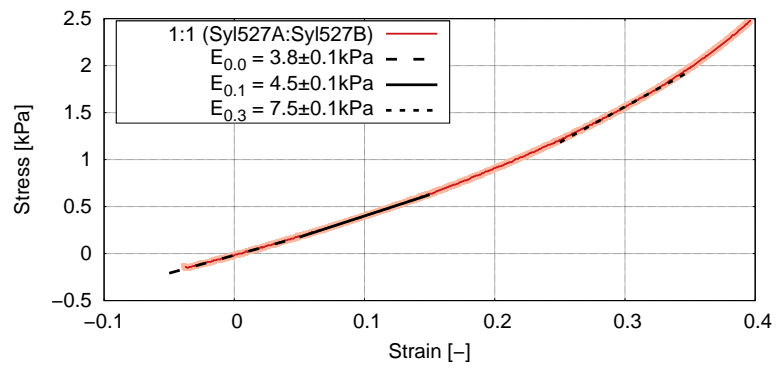

Figure 6. Deformation behaviour of 1.0 to 1.0 (Sylgard 527A to Sylgard 527B) mixture 


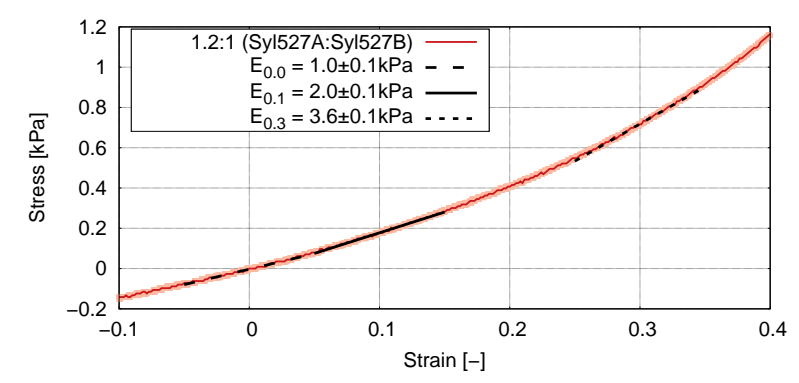

Figure 7. Deformation behaviour of 1.2 to 1.0 (Sylgard 527A to Sylgard 527B) mixture

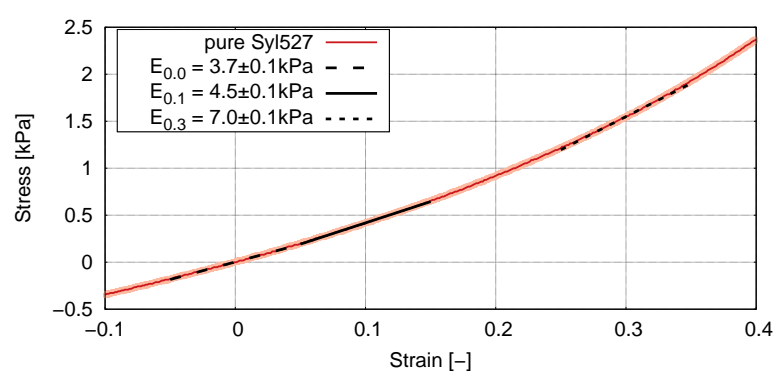

FIGURE 8. Deformation behaviour of pure Sylgard 527

\section{Conclusions}

To find optimised biocompatible PDMS substrates for cardiomyocytes developed for further mechanobiological studies the bulk material with different ratio of Sylgard 184, Sylgard 527A, and Sylgard 527B components. Mechanical analysis of soft PDMS require employment of custom designed precise loading device measuring the forces and displacement in millinewtons and micrometers respectively. From uniaxial mechanical test deformation behaviour and elastic properties was derived. Reliability of the instrumentation and measurement procedure for hyperelastic materials with Young's modulus from single kilopascal corresponding to desired values for further organ-ona-chip systems application [7] was successfully tested . For further analysis focused on dynamic testing simulating physiological processes new voice coil based loading device will be developed for cyclic loading of proposed materials with frequency up to $5 \mathrm{~Hz}$.

\section{ACKNOWLEDGEMENTS}

The research has been supported by the European Regional Development Fund in frame of the projects Kompetenzzentrum MechanoBiologie (ATCZ133) in the Interreg V-A Austria - Czech Republic programme.

\section{REFERENCES}

[1] J. Raczkowska, S. Prauzner-Bechcicki, J. Lukes, et al. Physico-chemical properties of pdms surfaces suitable as substrates for cell cultures. Applied Surface Science 389:247-254, 2016. DOI:10.1016/j.apsusc.2016.07.009

[2] J. Rihova, T. Suchy, L. Vistejnova, et al. Mechanical and structural properties of collagen nanofribrous layers under simulated body conditions. In $1^{\text {nth }}$ Youth Symposium On Experimental Solid Mechanics, vol. 25 of Acta Polytechnica CTU Proceedings. 2019. DOI:10.14311/APP.2019.25.0073.

[3] D. Kytyr, P. Zlamal, P. Koudelka, et al. Deformation analysis of gellan-gum based bone scaffold using on-the-fly tomography. Materials and Design 134:400-417, 2017. DOI:10.1016/j.matdes.2017.08.036

[4] R. Palchesko, L. Zhang, Y. Sun, A. Feinberg. Development of polydimethylsiloxane substrates with tunable elastic modulus to study cell mechanobiology in muscle and nerve. PLoS ONE 7(12), 2012. DOI:10.1371/journal.pone.0051499

[5] T. Fila, J. Sleichrt, D. Kytyr, et al. Deformation analysis of the spongious sample in simulated physiological conditions based on in-situ compression, $4 \mathrm{~d}$ computed tomography and fast readout detector. Journal of Instrumentation 13(11), 2018. DOI:10.1088/1748-0221/13/11/C11021.

[6] V. Rada, T. Fila, P. Zlamal, et al. Multi-channel control system for in-situ laboratory loading devices. In $16^{\text {th }}$ Youth Symposium On Experimental Solid Mechanics, vol. 18 of Acta Polytechnica CTU Proceedings, pp. 15-19. 2018. DOI:10.14311/APP.2018.18.0015

[7] S. Kratz, C. Eilenberger, P. Schuller, et al. Characterization of four functional biocompatible pressure-sensitive adhesives for rapid prototyping of cell-based lab-on-a-chip and organ-on-a-chip systems. Scientific Reports 9(1), 2019. DOI:10.1038/s41598-019-45633-x. 